\title{
Erratum
}

\section{Methods for fast analysis of anthocyanins and anthocyanidins in red wine}

\section{Karin Datzberger, Ingrid Steiner, Josef Washüttl, and Gerhard Kroyer}

Institute of Food Chemistry and Technology, Technical University Vienna, Getreidemarkt 9, A-1060 Vienna, Austria

In the original paper, the analysis of 3,5-diglucosides of anthocyanins was mentioned. It should have been: glucosides with 3,5-diglucosides as standard substances. As described, the anthocyanidin chlorides were detected after splitting the anthocyanins with hydrochloric acid. 